\title{
Effective treatment with tacrolimus for patients with non-severe aplastic anemia refractory/ intolerant to cyclosporine A: a retrospective study
}

Yali Du

Peking Union Medical College Hospital

Yuzhou Huang

Peking Union Medical College Hospital

Wenzhe Zhou

Peking Union Medical College Hospital

Xinjian Liu

Henan Cancer Hospital

Fangfei Chen

Peking Union Medical College Hospital

Chen Yang

Peking Union Medical College Hospital

Miao Chen

Peking Union Medical College Hospital

Jing Ruan

Peking Union Medical College Hospital

Bing Han ( $\square$ hanbingtg123@163.com )

Peking Union Medical College Hospital

Research article

Keywords: non-severe aplastic anemia; tacrolimus; cyclosporine A.

Posted Date: July 29th, 2020

DOl: https://doi.org/10.21203/rs.3.rs-41969/v1

License: (a) (1) This work is licensed under a Creative Commons Attribution 4.0 International License. Read Full License 


\section{Abstract}

Background: For the symptomatic non-severe aplastic anemia (NSAA) patients who cannot afford antithymocyte globulin (ATG) or allogeneic hematopoietic stem cell transplantation (HSCT), tacrolimus (FK) was occasionally reported to be an option if they were not response or tolerant to the cyclosporine $A$ (CsA).

Method: We collected and analyzed respectively 101 NSAA patients refractory or intolerant to CsA with no chance of HSCT or ATG, and treated them with tacrolimus for at least 6 months and followed up for at least one year.

Results: The overall response (ORR) was 38.6\% (complete response: $9.9 \%$; partial response: $28.7 \%$ ) and the median time to optimal response was $6(3 \otimes 10)$ months. $32(31.7 \%)$ cases had elevated creatinine level. 8 (7.9\%) cases had elevation of AST/ALT. 25.6\% (10/39) of patients relapsed at the end of follow-up. Age $(P=0.0005)$, FK concentration (4.0 12ng/ml, $P=0.0005)$ and intolerance to $C s A(P=0.012)$ were the independent risk factors for ORR. Treg cells level pre-FK treatment was much lower than healthy controls ( $3.7 \pm 0.6 \%$ vs $6.8 \pm 0.7 \%, P=0.0004)$, but increased significantly after $F K$ treatment $(3.7 \pm 0.6 \%$ vs. $7.1 \pm 0.8 \%$, $\mathrm{P}=0.0039)$.

Conclusion: Our data provide a possibility of tacrolimus as a salvage treatment for patients with NSAA refractory or intolerant to CsA.

\section{Background}

Aplastic anemia (AA) is a syndrome of hematopoietic failure from various reasons which lead to diminished or absent hematopoietic precursors in the bone marrow and attendant pancytopenia. For patients who need to be treated, age, disease severity, donor availability, and performance status play important roles in decision making[1, 2]. For patients with acquired severe AA (SAA), young patients ( $\leq 50 \mathrm{y}$ ) with HLA matched sibling donor should undergo allogeneic hematopoietic stem cell transplantation (HSCT) while older patients (50 years or older) and young patients without matched donor may receive full-dose immunosuppressive therapy (IST)- horse/rabbit anti-thymocyte globulin (ATG) combined with cyclosporine $\mathrm{A}(\mathrm{Cs} \mathrm{A})$ as the first line choice.

For those who do not reach the severe criteria (non-SAA, NSAA), and become transfusion dependent, ATG + cyclosporine A can be the first line therapy[1]. It has been demonstrated that the combination of ATG and CSA is superior to CSA alone for patients with NSAA[2], but in the real world, patients with NSAA were often treated with CsA alone due to the high costs and potential risks of ATG, especially in the developing counties. However, some patients do not response to CsA or relapse at the later stage of follow-up[3, 4]. On the other hand, patients may also withdraw from CsA due to some severe side effects, especially kidney impairment. 
Tacrolimus (FK)[5], an immunosuppressive agent similar to cyclosporine A, can inhibit the production and release of TNF-a, IFN- - , and interleukin-II (IL-2), and inhibits IL-2 induced activation of resting Tlymphocytes, had been used for prevention of graft-versus-host disease区GVHD) and other autoimmune diseases[6]. With 10冈100 times immunosuppressive effect than that of cyclosporine $A$ and less side effects, tacrolimus had been occasionally reported to treat AA patients refractory to CsA[7-10].

In this work, data from 101 patients with NSAA who were either refractory or intolerance to CsA, and were switched to the FK treatment for at least 6 months, were analyzed. The efficacy and safety of FK was evaluated.

\section{Materials And Methods}

\section{Patients selection}

From January 2017 to January 2019, all patients diagnosed as NSAA[11] and were treated with FK for at least 6 months in Peking Union Medical College Hospital (PUMCH) with relative integrated clinical data were reviewed retrospectively. Patients had to meet the following criteria for the final analysis: 1) 12 years or older ; 2) with confirmed diagnoses of NSAA; $3 \llbracket$ had been excluded for inherited bone marrow failure syndrome; 4) did not response after at least 6 months of standard CsA (3囚5mg/kg/d) treatment, or can't tolerate CsA due to the side effects before FK; 5) met at least one of the followings but not reach the criteria for SAA before FK treatment: hemoglobin $\leq 90 \mathrm{~g} / \mathrm{L}$, or neutrophil (ANC) $\leq 0.8 \times 10^{9} / \mathrm{L}$, or platelet counts $\leq 30 \times 10^{9} / \mathrm{L} ; 6$ ) with no history of HSCT. 7) not ATG (could not afford or were not willing to) or allo-HSCT candidate; 8) Scr $<200 \mu \mathrm{mol} / \mathrm{L}$ prior FK treatment.

\section{Treatment regimen}

All patients had been treated with tacrolimus (Cyfokei, Hangzhou Zhongmei Huadong Pharmaceutical Co, Ltd) at initial dose of $1 \mathrm{mg}$ bid, and adjusted the dosage to maintain the plasma concentration of $4 \mathbb{Q} 10$ $\mathrm{ng} / \mathrm{mL}$ for at least 6 months. Patients with renal insufficiency before FK or after FK had to reduce drug dose, therefore, they may not reach the standard plasma concentration. Patients who reacted to FK continued the treatment for at least 1.5 years and tapered gradually afterwards. The longest time exposed to FK was 30 months. Effectiveness of FK was evaluated after at least 6 months of treatment and patients did not reach PR after 6 months were recognized as no response (NR) and stop FK treatment but the follow up continued till the end of the study. Patients received transfusions as supportive care to maintain platelets at a level above $10 \times 10^{9} / \mathrm{L}$ and hemoglobin at a level above $60 \mathrm{~g} / \mathrm{L}$.

\section{Clinical evaluations}

Patients were recorded with symptoms (including side effects), signs and laboratory assessments (complete blood count with differential and serum chemistry profile, ECG or other tests if needed) and plasma FK concentration at the time of one months, three months, six months, twelve months after 
treatment and at the end of follow-up. All the medical records were taken from the official documents from our hospital. RBC or platelet transfusion requirements were also checked before and after FK treatment.

Criteria for effectiveness were: complete response (CR): ANC >1.5 × 109/L, hemoglobin $>110 \mathrm{~g} / \mathrm{L}$ and platelet counts $>100 \times 109 / \mathrm{L}$ for 2 months. Partial response (PR): patients who met response criteria for one or more lineages at 12 weeks but did not meet the criteria of CR: platelet response was defined as an increase of $20 \times 109 / L$ or more above the baseline value, or independence from platelet transfusions for a minimum of eight weeks in patients who were previously transfusion-dependent; erythroid response was defined as an increase in the hemoglobin level by $15 \mathrm{~g} / \mathrm{L}$ or more without transfusion of packed red cells or a reduction in the number of units of packed red cells transfused by at least four units for eight consecutive weeks, as compared with transfusion requirements during the eight weeks preceding FK treatment; neutrophil response was defined as an absolute increase in the neutrophil count of more than $0.5 \times 109 / \mathrm{L}$ in patients with a pretreatment count of less than $0.5 \times 109 / \mathrm{L}$, or at least a $100 \%$ increase over the baseline neutrophil count. NR: didn't have any of the above responses.

Patients were followed up for at least one year or till the time of death. The final outcome was recorded either from the medical documents or telephone interview of the patients or their relatives. Adverse events (AE) were evaluated according to the National Cancer Institute Common Toxicity Criteria for Adverse Events (CTCAE version 4.0).

\section{Examination of regulatory $\mathrm{T}$ cells}

Anti-CD 4 conjugated to FITC, anti-foxP3 conjugated to APC and anti-CD25 conjugated to PE antibodies was obtained from BD biosciences (New Jersey, USA). The regulatory $T\left(T_{\text {reg }}\right)$ cells were determined by staining with markers of CD4-FITC, CD25-PE and fox $\mathrm{P}_{3}-\mathrm{APC}$, and subsequently analyzed by a Beckman Coulter FC500 flow cytometry (California, USA). Total events of 50000 were gated based on forward (FSC) and side-scatter (SSC) characteristics and dot plots for $T_{\text {reg }}$ cells were gated on CD4+cells. $T_{\text {reg }}$ cells were defined as $C D 4+C D 25+f_{0 x} P_{3}+$ co-expression and expressed as a percentage of total CD4+ T population[12, 13]. Only 31 patients gave their consent to measure $T_{\text {reg }}$ cells before and after $F K$ treatment. In the meantime, we recruited eight age and gender matched healthy volunteers and tested their $\mathrm{T}_{\text {reg }}$ cells as normal controls.

\section{Statistical analysis}

Proportions were given for categorical variables, mean \pm standard deviation was given for continuous variables, and median (minimum, maximum) were given for variables that were not normally distributed. Chi-square tests fisher's exact test were used for categorical measures. Logistic regression models were used to estimate the odds ratio (OR) and $95 \%$ confidence interval $(\mathrm{Cl})$ for the association between potential risk factors and ORR. All analyses were conducted using SAS version 9.4 (SAS Institute, Inc, Cary, NC, USA). A P-value $<0.05$ was considered statistically significant. 
The median values and frequencies for the categorical data were described by descriptive analyses. SPSS 24.0 software (IBM, NY, USA) was used for statistical analysis. Data were analyzed by Fisher's exact test and Pearson's chi-squared test. $\mathrm{P} \otimes 0.05$ was considered as statistically significant.

\section{Results}

\section{Patient characteristics}

From January 2017 to January 2019, there were altogether 156 NSAA patients treated with FK. 55 patients were excluded either due to lost follow-up (32 patients), withdrawal of FK (12 patients) within 6 months after FK, follow-up less than 12 months (10 patients) or denying the consent form (3patients). There were 101 patients enrolled for the final analysis, including 49 males (48.5\%) and 52 females (51.5\%), with a median age of 46 (range 14『83) years. Among them, 58 (57.4\%) patients were under the age of 50-yearold. PUMCH is known for difficult and complicated cases so most of the refractory NSAA are referred to our clinic which make our cohort highly selective-about $20 \%$ of younger patients discontinue CsA due to renal disfunction after repeating use of CsA in long period of time. Before FK treatment, patients' hematological and biochemistry parameters were as shown in Table 1. As for the chromosome abnormalities, +8 was found in 5 patients, $-Y$ in 3 patients, 20q- in 2 patient and monosomy 7 in 1 patient. 7 patients had notable PNH clone邓percentage of flaer negative neutrophil from 3.5\% $18.3 \%) .50$ (48.1\%) patients were red blood cell transfusion dependent and the median transfusion requirements were 6 (range $4 \rrbracket 10)$ units/eight weeks and $26(25.0 \%)$ patients were platelet transfusion dependent and the median transfusion requirements were 4 (range 1ه5) units/eight weeks before FK treatment (Table 1) .

\section{Table 1 Patients' baseline demographic and clinical characters}




\begin{tabular}{|c|c|}
\hline Baseline characters & $\mathrm{n}=101$ \\
\hline Age (year) median (min,max) & $46(14,83)$ \\
\hline \multicolumn{2}{|l|}{ Sex } \\
\hline Male $\mathrm{n}(\%)$ & 49 (48.5) \\
\hline Female n (\%) & $52(51.5)$ \\
\hline Hemoglobin $(\mathrm{g} / \mathrm{L})$ mean $(\mathrm{SD})$ & $61.88(28.13)$ \\
\hline Reticulocyte $\left(10^{12} / \mathrm{L}\right)$ mean (SD) & $6.13(10.01)$ \\
\hline White cell count $\left(10^{9 /} \mathrm{L}\right)$ mean $(\mathrm{SD})$ & $3.08(1.03)$ \\
\hline Neutrophil count $\left(10^{9} / \mathrm{L}\right)$ mean (SD) & $1.32(0.80)$ \\
\hline Platelet $\left(10^{9} / \mathrm{L}\right)$ mean $(\mathrm{SD})$ & $38.26(62.50)$ \\
\hline SGPT (U/L) mean (SD) & $26.88(24.48)$ \\
\hline $\operatorname{Scr}(\mu \mathrm{mol} / \mathrm{L})$ mean $(\mathrm{SD})$ & $98.56(58.66)$ \\
\hline $\mathrm{SF}(\mu \mathrm{g} / \mathrm{L})$ mean $(\mathrm{SD})$ & $1761.23(1937.18)$ \\
\hline Glu (mmol/L) mean (SD) & $6.51(3.97)$ \\
\hline Notable PNH clone $\mathrm{n}(\%)$ & $7(6.9)$ \\
\hline $\mathrm{RBC}$ transfusion dependent $\mathrm{n}(\%)$ & $50(49.5)$ \\
\hline Platelet transfusion dependent n (\%) & $26(25.7)$ \\
\hline \multicolumn{2}{|l|}{ Chromosome abnormalities } \\
\hline$+8 \mathrm{n}(\%)$ & $5(5.0)$ \\
\hline$-Y$ n (\%) & $3(3.0)$ \\
\hline $20 q-n(\%)$ & $2(2.0)$ \\
\hline Monosomy 7 n (\%) & $1(1.0)$ \\
\hline
\end{tabular}

Patients had been treated with CsA alone before FK treatment. Among them, 45 patients (refractory patients) had no response and the median time for CsA treatment was 8 (6囚10) months, 36 patients (relapsed patients) relapsed when CsA was tapered or stopped but did not response when CsA were added again or dosage increased and the median time for CsA treatment was $18 \otimes 12 \varangle 60 \rrbracket$ months. 20 patients (intolerant patients) could not tolerate CsA due to severe side effects like gingival hyperplasia, muscle tremor, kidney impairment, gastrointestinal disturbance, etc. The median time of CsA exposure for those patients was 4 (3囚7) months. None of them had reached PR before FK treatment. Of all patients, 
$50(53.2 \%)$ patients had FK concentration of $4 \llbracket 12 \mathrm{ng} / \mathrm{mL}$ within three months of tacrolimus treatment, while others did not reach the aim concentration due to the kidney limitation.

\section{Efficacy}

The median follow-up time was 19 (14囚36) months since FK started. At the end of follow-up, the median time on FK treatment was 14 (6区30) months. Of all the 101 patients, the median time to response (at least partial response, PR) was 4 (1区6) months, the medium time to optimal response was 6 (3囚10) months. There was 10 (9.9\%) CR, 29 (28.7\%) PR, 62 (61.4\%) NR, with OR (CR+PR) rate of 38.6\%.

Of the 39 patients who achieved OR, $9 \varangle 23.1 \% \bigotimes$ were solely erythroid response ( 6 of them became transfusion independent), $11 \varangle 28.2 \% \bigotimes$ were solely platelet response , $3 \varangle 7.7 \% \varangle$ with neutrophil response, 9 (23.1\%) patients had bilineage responses and $7(17.9 \%)$ patients had trilineage response.

Moreover, 14 patients with liver or kidney impairment improved their liver and kidney function after FK treatment, among them 10 patients with elevated creatinine level and the other 4 patients with abnormal bilirubin level prior FK treatment returned to normal after 6 months of medication. 10 patients' gingival hyperplasia and gastrointestinal symptoms were significantly improved.

Subgroup analysis showed that the OR rate (ORR) for patients younger than 50 years old was $50.0 \%$, significantly higher than that of patients over 50 years old $(23.3 \%, P=0.004)$. Patients with FK concentration $4.0 \otimes 12.0 \mathrm{ng} / \mathrm{ml}$ had higher ORR (49.2\%) compared with those with concentration $\varangle 4.0 \mathrm{ng} / \mathrm{ml}$ $(22.7 \%, P=0005)$. Females had higher ORR than males $(P=0.0442)$. There was no significant difference in ORR between patients with refractory/relapsed and intolerant to CsA (35.8\% vs 50.0\%, $\mathrm{P}=0.2429$, Table 2$)$. However, multiple logistic regression result indicated that age $(P=0.0005) \otimes F K$ concentration $(4.0 \sim 12 \mathrm{ng} / \mathrm{ml})(\mathrm{P}=0.0005)$ and intolerance to $\mathrm{CsA}(\mathrm{P}=0.0142)$ were the independent risk factors for ORR (Table 3).

\section{Table 2 Patients' response in different groups}

\begin{tabular}{|c|c|c|c|c|c|c|c|c|}
\hline & & Number & $\mathrm{CR}(\mathrm{N} / \%)$ & $\begin{array}{c}\mathrm{P}^{*} \\
\text { value }\end{array}$ & $\mathrm{PR}(\mathrm{N} / \%)$ & $\begin{array}{c}\mathrm{P}^{\&} \\
\text { value }\end{array}$ & ORR(N/\%) & $\begin{array}{c}\mathrm{P}^{\#} \\
\text { value }\end{array}$ \\
\hline \multirow[t]{2}{*}{ Gender } & Male & 49 & $2(4.1)$ & 0.09338 & $12(24.5)$ & 0.3625 & $14(28.6)$ & 0.0442 \\
\hline & Female & 52 & $8(15.4)$ & & $17(32.7)$ & & $25(48.1)$ & \\
\hline \multirow{2}{*}{$\begin{array}{l}\text { Age } \\
\text { (range)years }\end{array}$} & $\leq 50$ & 58 & $6(10.3)$ & 1 & $23(39.7)$ & 0.0033 & $29(50.0)$ & 0.004 \\
\hline & $\square 50$ & 43 & $4(9.3)$ & & $6(14.0)$ & & $10(23.3)$ & \\
\hline \multirow[t]{2}{*}{$\mathrm{CsA}$} & refractory/relapsed & 81 & $7(8.6)$ & 0.4103 & $22(27.2)$ & 0.4877 & $29(35.8)$ & 0.2429 \\
\hline & intolerant & 20 & $3(15.0)$ & & $7(35.0)$ & & $10(50.0)$ & \\
\hline \multirow{2}{*}{$\begin{array}{l}\text { FK } \\
\text { concentration }\end{array}$} & $<4.0 \mathrm{ng} / \mathrm{ml}$ & 44 & $2(4.5)$ & 0.0977 & $8(18.2)$ & 0.0126 & $10(22.7)$ & 0.0005 \\
\hline & $4.0 \sim 12 \mathrm{ng} / \mathrm{ml}$ & 50 & $8(13.6)$ & & 21(35.6) & & $29(49.2)$ & \\
\hline
\end{tabular}


*P value indicate the comparison of $\mathrm{CR}$ among different groups, \&P value indicate the comparison of $\mathrm{PR}$ among different groups, \#P value indicate the comparison of ORR among different groups

Table 3 Multivariate analysis for the factors associated with ORR

\begin{tabular}{ccccccc}
\hline & Coefficient & $\begin{array}{c}\text { Standard } \\
\text { error }\end{array}$ & OR & $\begin{array}{c}\text { lower bound } \\
\text { 95\% CI }\end{array}$ & $\begin{array}{c}\text { upper bound } \\
95 \% \text { CI }\end{array}$ & P \\
\hline Male(ref=Female) & -0.7727 & 0.5187 & 0.462 & 0.167 & 1.276 & 0.1363 \\
Age (year) & -0.0660 & 0.0191 & 0.936 & 0.902 & 0.972 & 0.0005 \\
\hline FK $(4 \sim 12 \mathrm{ng} / \mathrm{ml})$ & 1.9729 & 0.5636 & 7.192 & 2.383 & 21.706 & 0.0005 \\
$\begin{array}{c}\text { refractory/relapse } \\
\text { (ref=intolerant) }\end{array}$ & -1.7391 & 0.7090 & 0.176 & 0.044 & 0.705 & 0.0142 \\
\hline
\end{tabular}

\section{Safety}

In a total of 101 patients, there were $32(31.7 \%)$ cases of elevated creatinine level ( 9 of them had increased Scr level before FK treatment), 8 cases (7.9\%) with elevation of AST/ALT, 6 (5.9\%囚cases of hypertension, 5 (5.0\%) cases of elevated bilirubin, and 2 (2.0\%) cases of drug allergy, most of them were grade I-II in CTCAE criteria 4.0 which recovered after symptomatic treatment. Severe adverse events resulting in drug withdraw or hospitalization included the followings: 4 cases of grade III creatinine increase, 4 episodes of fever with grade IIIIIV neutropenia, one of which linked to culture-confirmed infection, occurred in one patient who did not have a response.

\section{Relapse, survival and clonal evolution}

For the 39 patients who had reached CR or PR, 25.6\% (10/39) patients relapsed at the median of 12 (10区 16) months before FK tapering. Clonal evolution to acute myeloid leukemia (AML) was observed in one patient with monosomy 7 who did not response to FK. Two patients who did not response evolved into myelodysplastic syndrome (MDS) one year after treatment and lived with supportive care. No increase of $\mathrm{PNH}$ clone, nor clonal evolution to MDS or AML developed in other patients in our cohort. Four young patients who did not response underwent HSCT and achieved CR.

There were two deaths during the follow-up period in the no response cohort, one was due to severe infections as mentioned above, and the other was the patient transforming to AML who died of infections 2 months after chemotherapy. Other patients with no response lived with supportive care. No other deaths were observed in the cohort.

\section{Regulatory T cells in peripheral blood before and after FK}

Thirty-one patients had tested Treg cells before and after FK, and they all achieved at least PR and the blood was taken after six months of treatment, meanwhile the level of $\mathrm{T}_{\text {reg }}$ cells from eight age and sexmatched normal volunteers was taken as controls. Level of $T_{\text {reg }}$ cells pre $F K$ was much lower compared with that of healthy controls ( $\mathrm{T}_{\text {reg }} / \mathrm{CD} 4+\mathrm{T}: 3.7 \pm 0.6 \%$ vs $\left.6.8 \pm 0.7 \%, P=0.0004\right)$. As expected, the level of $\mathrm{T}_{\text {reg }}$ 
cells increased significantly after FK treatment $\left(T_{\text {reg }} / C D 4+T\right.$, before: $3.7 \pm 0.6 \% v s$. after: $7.1 \pm 0.8 \% \rrbracket$ $P=0.0039$, Fig 1).

\section{Discussion}

Although CsA has achieved $40 \sim 50 \%$ of response rate and has long-term effect maintainance and survival for patients with non-severe aplastic anemia[2, 3], there is still an unmet need when patients do not response, or relapse, or intolerant to CsA[8, 14]. Although intensive immunosuppressive therapy with ATG, or even HSCT can further improve the response rate, these treatments are relatively expensive, risky, sometimes unavailable, and not suitable to old and weak patients[15]. Eltrombopag, an oral thrombopoietin receptor agonist[16], launched in China recently, has emerged as a promising new drug for the treatment of aplastic anemia[17, 18]. But the price is too high for the regular use for most of the patients.

Our study provided a large cohort with relatively long-term follow-up for tacrolimus' second line use in NSAA. Many patients in our study were referred from the local hospitals who had been refractory or intolerant to the CsA treatment. According to our study, at the median time of 4 (1ष6) months, $38.6 \%$ patients achieved response to various degree, with $9.9 \% \mathrm{CR}$ and $28.7 \% \mathrm{PR}$. Of the patients who achieved $\mathrm{CR}$ and $\mathrm{PR}$, most of them had monolineage reaction, a few had bilineage or trilineage response. It seemed that for those patients, FK can have some reaction, but mainly with PR rather than CR. Patients reached the optimal effects at the medium of 6 months. $25.6 \%$ of patients who responded relapsed at the median of 12 months before FK tapering. Even though, patients may have some benefit from hematology improvement and getting rid of transfusion since most of the patients in our study were CsA resistant or intolerant, FK provided a different choice for them with acceptable response.

Most of the studies on tacrolimus so far are either case reports, or focus on replacing CsA in ATG or bone marrow transplantation (BMT) regimens[6-8, 14]. However, those studies have verified our results to some extent. Matched-pair analysis by Yagasaki $\mathrm{H}$ et al[6] to compare FK/MTX with CsA/MTX in patients with SAA who received U-BMT囚Unrelated BMT ¿indicated that the 5-year survival rate was higher in FK group compared with the CsA group, showing the superiority of FK/MTX over CsA/MTX in overall survival because of the lower incidence of transplantation-related deaths. Another study finished by Zhu X et al[7] showed that a total of $54 \%$ of the 13 patients with SAA in the tacrolimus + rabbit-ATG group and $42 \%$ of the 24 cases in the ATG + CsA group achieved the criteria for CR, and the PR rate was similar between the two groups, providing a possibility of using tacrolimus as part of the IST regimen. The effect of tacrolimus has been further manifested in some case reports that FK can improve the hematopoiesis of some AA patients refractory to CsA, and pediatric study that tacrolimus could be used in the maintenance phase of IST with $88 \%$ of $\mathrm{CR}$ in SAA[8].

More important, tacrolimus seemed to be safer and more tolerable compared with CsA: a large amount of patients with SAA treated with CsA showed hirsutism and gingival hyperplasia, and kidney injury was very commonly seen[14]. In our cohort, there were $31.7 \%$ cases of elevated creatinine level, probably due to the 
long-term use of CsA before FK treatment, but most of them were mild and controllable (four patients withdrew FK due to grade III creatinine increase). Very few cases of elevated AST/ALT, hypertension, elevated bilirubin and drug allergy. No hirsutism or gingival hyperplasia was noticed. Besides, 14 patients with liver or kidney impairment improved their liver and kidney function after FK treatment. These results were in consistence to what had been reported previously that tacrolimus has exhibited much more safety and less impact on renal function and blood pressure than cyclosporine $A[19,20]$.

We further analyzed the possible influence factors for FK response. Patients with old age seemed to have poorer reaction compared with those with younger age, similar findings have been reported in patients with SAA who received either ATG+CsA or allo-BMT[2]. Older patients may probably have less remaining hematopoiesis and do not have enough storage of kidney function for proper FK concentration, which may contribute to the poorer response. Not surprisingly, patients with different FK concentration had different ORR, indicating the importance of maintaining enough drug expose. Even though, part of patients with renal insufficiency could not receive full dose of FK and had to stay with low concentration, and the ORR was lower for this part of patients accordingly. In our cohort, those who were refractory/relapsed to CsA were inferior in ORR as compared with those intolerant to CsA in multiple logistic regression analysis. It is easily understood that patients who stopped CsA due to side effects might have more chance of response if they are treated continuously with FK as compared with those had no response to CsA at all. Although female gender played a role in the univariate analysis, logistic regression indicated that it was not the independent risk factor.

Previous research has demonstrated that IL-2 can enhance the proliferation and expression of TNF-a of CD8(+)HLA-DR(+)T cells from AA patients and such effect can be inhibited by tacrolimus, which is also true for CsA[21, 22]. However, tacrolimus may have different way of action from that of CsA because we observed the decrease level of $T_{\text {reg }}$ cells after CsA treatment was significant improved after full dose of tacrolimus in patients who responded[13,23]. It is well known that $T_{\text {reg }}$ cells, as marked by CD4+CD25+ and Foxp3, is obviously lower in patients with untreated AA compared with normal people and increase significantly after effective treatment with IST[24, 25].

Three patients had clonal evolution to either MDS or AML, which was similar to what had been reported in patients refractory to CsA[26]. Patients who did not react to immunosuppressive agent either had greater pressure of clonal selection under the bone marrow failure environment or had more clonal hematopoiesis at the very beginning. For those who had no response, infection and bleeding were common, 1 patient died of infection at the time of 12 months after FK treatment, which were also seen in patients failed from other treatments.

There are still some limitations of the study. $1 / 3$ of patients had been excluded for analysis due to short period of treatment or follow-up time, which may cause bias because part of them may have less positive response to FK. Follow-up time was limited for long term efficacy, survival and clone evolution. No regular molecular tests were done in our cohort before and after FK treatment which may limit the prediction of clone evolution. 


\section{Conclusion}

Even though, we summarized the second-line use of FK in the real world for the first time. As shown above, FK can be an alternative selection for those who are refractory, or intolerance to CsA with an acceptable price and tolerance. Further investigation with larger patients' number and proper control is needed.

\section{List Of Abbreviations}

NSAA: non-severe aplastic anemia

SAA: severe aplastic anemia

AA: aplastic anemia

ATG: Anti-thymocyte globulin

HSCT: hematopoietic stem cell transplantation

FK: tacrolimus

CsA: cyclosporine A

ORR: overall response rate

CR: complete response

PR: partial response

NR: no response

OR: overall response

IST: immunosuppression therapy

GVHD: graft-versus-host disease

PUMCH: Peking Union Medical College Hospital

$\mathrm{AE}:$ adverse events

CTCAE: Common Toxicity Criteria for Adverse Events

Treg cells: regulatory $\mathrm{T}$ cells

Cl: confidence interval

AML: acute myeloid leukemia 
MDS: myelodysplastic syndrome

\section{Declarations}

\section{Ethics approval and consent to participate}

Written informed consent was obtained from all patients before the data were collected and all the clinical procedure was in accordance with the Declaration of Helsinki and was approved by the ethnic committee of Peking Union Medical Colleague Hospital.

\section{Consent for publication}

Consent for publication were got from all patients accompanied with informed consent at the beginning of the study.

\section{Availability of data and materials}

The datasets used and/or analysed during the current study are available from the corresponding author on reasonable request.

\section{Competing interests}

The authors declare that they have no competing interests.

\section{Funding}

This study was supported by grants from Beijing Natural Science Foundation (7192168), the Chinese Academy of Medical Sciences (CAMS) innovation fund for medical sciences (2016-12M-3-004) and the Non-profit Central Research Institute Fund of Chinese Academy of Medical Sciences (2019XK 320047).

\section{Authors' contributions}

Bing. $H$ designed the study. $Y L$ Du and $Y Z$ Huang analyzed clinical and experimental data and wrote the manuscript. WZ Zhou and XJ Liu helped to design the study and collected the samples. F Chen, $C$ Yang, $M$ Chen helped analyzed the risk model. All authors reviewed the manuscript finally. Bing. Happroved the final submission of the manuscript.

\section{Acknowledgements}

Not applicable.

\section{References}

1. Pierri, F. and C. Dufour, Management of aplastic anemia after failure of frontline immunosuppression. Expert Rev Hematol, 2019. 12(10): p. 809-819. 
2. Marsh, J., et al., Prospective randomized multicenter study comparing cyclosporin alone versus the combination of antithymocyte globulin and cyclosporin for treatment of patients with nonsevere aplastic anemia: a report from the European Blood and Marrow Transplant (EBMT) Severe Aplastic Anaemia Working Party. Blood, 1999. 93(7): p. 2191-5.

3. Zhu, X.F., et al., Current Treatment Patterns of Aplastic Anemia in China: A Prospective Cohort Registry Study. Acta Haematol, 2019. 142(3): p. 162-170.

4. Alashkar, F., et al., Immunosuppressive therapy (IST) in adult patients with acquired aplastic anemia (AA): A single-center experience over the past 15 years. Eur J Haematol, 2019. 103(1): p. 18-25.

5. Tong, J., et al., Effect of FK-506 and cyclosporin A on in vitro CFU-GM growth in severe aplastic anemia patients. Haematologica, 1992. 77(4): p. 369-70.

6. Yagasaki, H., et al., Tacrolimus/Methotrexate versus cyclosporine/methotrexate as graft-versus-host disease prophylaxis in patients with severe aplastic anemia who received bone marrow transplantation from unrelated donors: results of matched pair analysis. Biol Blood Marrow Transplant, 2009. 15(12): p. 1603-8.

7. Zhu, X., et al., Pilot study using tacrolimus rather than cyclosporine plus antithymocyte globulin as an immunosuppressive therapy regimen option for severe aplastic anemia in adults. Blood Cells Mol Dis, 2014. 53(3): p. 157-60.

8. Alsultan, A., et al., Tacrolimus as an alternative to cyclosporine in the maintenance phase of immunosuppressive therapy for severe aplastic anemia in children. Pediatr Blood Cancer, 2009. 52(5): p. 626-30.

9. Inamoto, Y., et al., Tacrolimus versus Cyclosporine after Hematopoietic Cell Transplantation for Acquired Aplastic Anemia. Biol Blood Marrow Transplant, 2015. 21(10): p. 1776-82.

10. Macartney, C., et al., Complete response to tacrolimus in a child with severe aplastic anemia resistant to cyclosporin A. Pediatr Blood Cancer, 2009. 52(4): p. 525-7.

11. Camitta, B.M., R. Storb, and E.D. Thomas, Aplastic anemia (second of two parts): pathogenesis, diagnosis, treatment, and prognosis. N Engl J Med, 1982. 306(12): p. 712-8.

12. Lin, S., et al., Roles of regulatory $T$ cells in the pathogenesis of pediatric aplastic anemia. Pediatr Hematol Oncol, 2019. 36(4): p. 198-210.

13. Dong, Q.E., et al., [Inhibitory effects of tacrolimus on effector $T$ cells from patients with severe aplastic anemia]. Zhonghua Yi Xue Za Zhi, 2013. 93(20): p. 1541-5.

14. Vallejo, C., P. Iniesta, and J.M. Moraleda, Resolution of cyclosporine-induced gingival hyperplasia resistant to azithromycin by switching to tacrolimus. Haematologica, 2001. 86(1): p. 110.

15. Tisdale, J.F., D.E. Dunn, and J. Maciejewski, Cyclophosphamide and other new agents for the treatment of severe aplastic anemia. Semin Hematol, 2000. 37(1): p. 102-9.

16. Fattizzo, B., et al., Eltrombopag in Immune Thrombocytopenia, Aplastic Anemia, and Myelodysplastic Syndrome: From Megakaryopoiesis to Immunomodulation. Drugs, 2019.

17. Ecsedi, M., et al., Use of eltrombopag in aplastic anemia in Europe. Ann Hematol, 2019. 98(6): p. 1341-1350. 
18. Cheng, H., et al., Eltrombopag combined with cyclosporine may have an effect on very severe aplastic anemia. Ann Hematol, 2019. 98(8): p. 2009-2011.

19. Liu, J.Y., et al., Tacrolimus Versus Cyclosporine as Primary Immunosuppressant After Renal Transplantation: A Meta-Analysis and Economics Evaluation. Am J Ther, 2016. 23(3): p. e810-24.

20. Muduma, G., et al., Systematic Review and Meta-Analysis of Tacrolimus versus Ciclosporin as Primary Immunosuppression After Liver Transplant. PLoS One, 2016. 11(11): p. e0160421.

21. Halloran, P.F. and J. Madrenas, The mechanism of action of cyclosporine: a perspective for the 90's. Clin Biochem, 1991. 24(1): p. 3-7.

22. Bai, Y.J., et al., [Regulatory function of tacrolimus and CSA on CD4/CD8 T lymphocyte subgroups and costimulators on them in allo-liver recipients]. Xi Bao Yu Fen Zi Mian Yi Xue Za Zhi, 2008. 24(10): p. 989-92.

23. Miroux, C., et al., In vitro effects of cyclosporine $A$ and tacrolimus on regulatory T-cell proliferation and function. Transplantation, 2012. 94(2): p. 123-31.

24. Solomou, E.E., et al., Deficient CD4+CD25+ FOXP3 + T regulatory cells in acquired aplastic anemia. Blood, 2007. 110(5): p. 1603-6.

25. Kordasti, S., et al., Deep phenotyping of Tregs identifies an immune signature for idiopathic aplastic anemia and predicts response to treatment. Blood, 2016. 128(9): p. 1193-205.

26. Hama, A., et al., Comparison of long-term outcomes between children with aplastic anemia and refractory cytopenia of childhood who received immunosuppressive therapy with antithymocyte globulin and cyclosporine. Haematologica, 2015. 100(11): p. 1426-33.

\section{Figures}


A
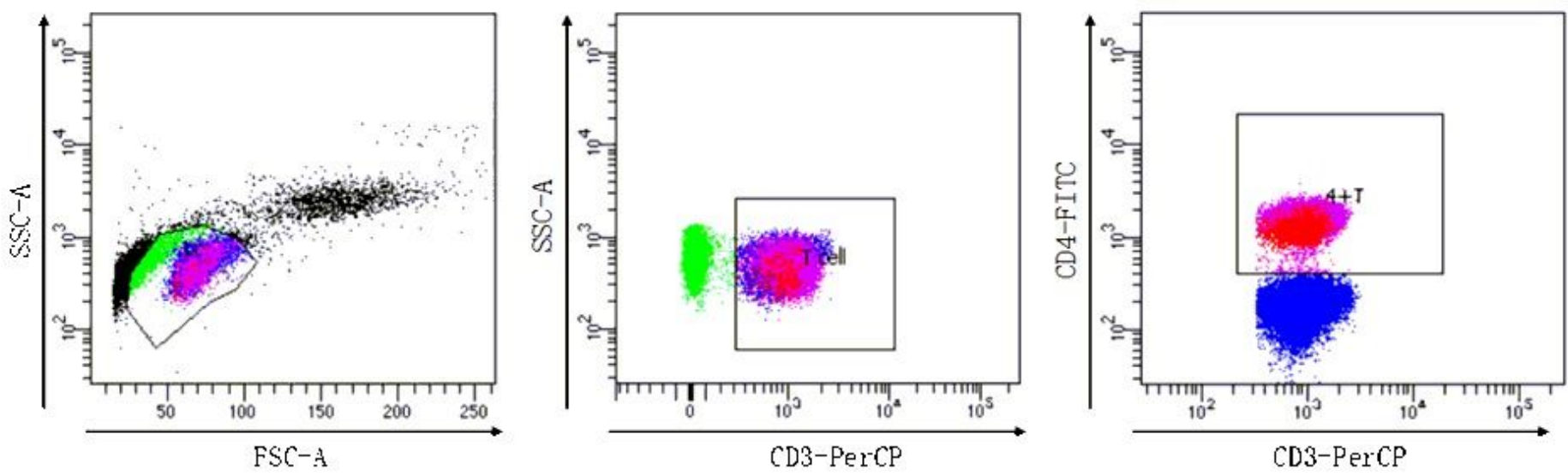

B

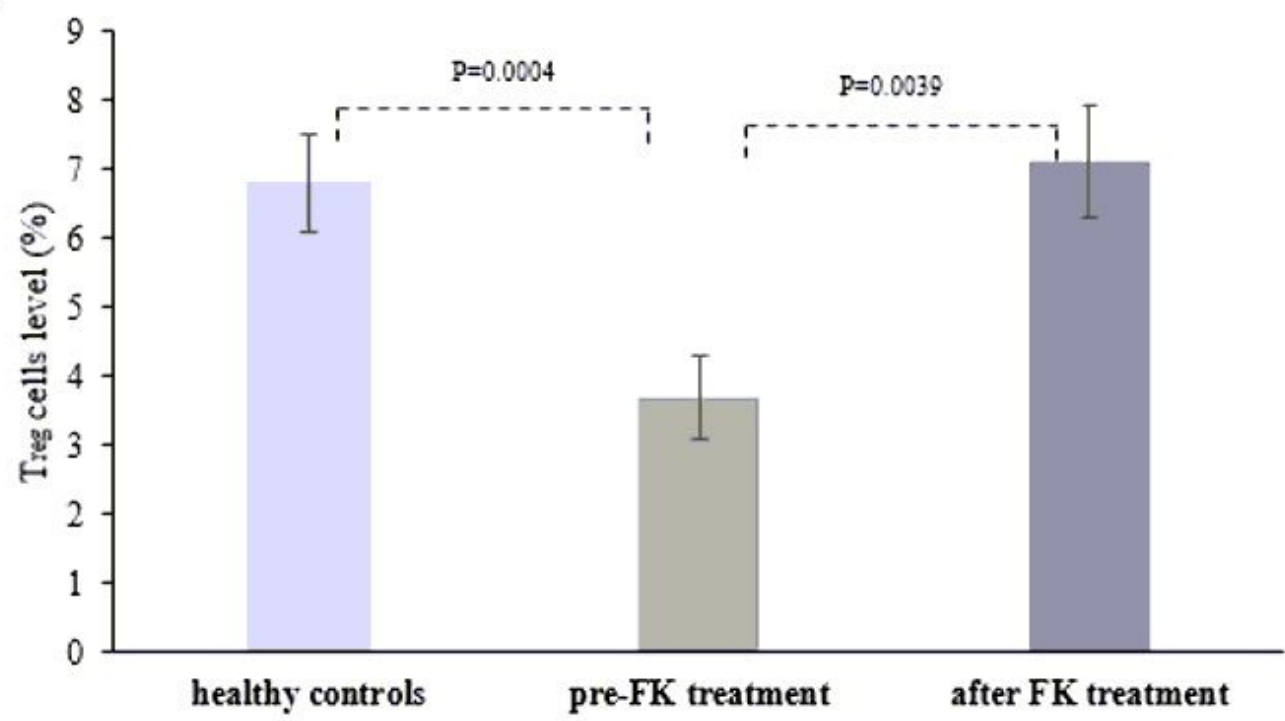

Figure 1

Regulatory T cells in peripheral blood before and after FK. A. Total events of 50000 were gated based on forward (FSC) and side-scatter (SSC) characteristics and dot plots for Treg cells were gated on CD4+cells. Treg cells were defined as CD4+CD25+foxP3+ co-expression and expressed as a percentage of total CD4+ $T$ population. B. The expression of Treg cells from 31 patients who had achieved at least PR was tested before and 6 months after FK treatment, eight age and sex-matched normal volunteers were taken as controls. Level of Treg cells pre FK was much lower compared to that of healthy controls, but increased significantly after FK treatment. 